\title{
Determinants of Survival After Emergency Intrapericardial Cisplatin Treatment in Cancer Patients with Recurrent Hemodynamic Instability After Pericardiocentesis
}

\author{
SZYMON DAROCHA ${ }^{1}$, MICHAŁ WILK ${ }^{1,2}$, ANNA WALASZKOWSKA-CZYŻ ${ }^{2}$, JAROSŁAW KĘPSKI ${ }^{1}$, \\ RAFAŁ MAŃCZAK ${ }^{1}$, MARCIN KURZYNA ${ }^{1}$, ADAM TORBICKI $^{1}$ and SEBASTIAN SZMIT ${ }^{1}$ \\ ${ }^{1}$ Department of Pulmonary Circulation, Thromboembolic Diseases and Cardiology, \\ Centre of Postgraduate Medical Education, and \\ ${ }^{2}$ Department of Clinical Oncology, European Health Centre, Otwock, Poland
}

\begin{abstract}
Background: Pericardial effusion is associated with high mortality in oncology. The etiology of infectious pericarditis and iatrogenic effects of previous radio/chemotherapy may be always suspected, especially when a subsequent episode is observed. Patients and Methods: The study included 17 hemodynamically-unstable patients with cancer due to recurrent pericardial bloody effusion after previous pericardiocentesis and analyzed survival determinants after intrapericardial chemotherapy with cisplatin. Results: The mortality rate was not significantly associated with the level of $N$-terminal pro-B type natriuretic peptide, low hemoglobin $(<12 \mathrm{~g} / \mathrm{dl})$, elevated white blood cell account $\left(>10^{4} / \mu \mathrm{l}\right)$, large volume $(>1500$ $\mathrm{ml}$ ) and long duration (>8 days) of pericardial drainage, cardiac arrhythmias, positive culture test results nor fever occurring during cisplatin administration. Subsequent systemic anticancer therapy was the strongest factor determining a longer survival (hazard ratio $(H R)=0.31,95 \%$ confidence interval $(C I)=0.11-0.9 ; p=0.03)$. Conclusion: Efficacy of rescue intrapericardial chemotherapy with cisplatin is independent of parameters of hemodynamic instability and levels of inflammatory markers in recurrent pericardial effusion.
\end{abstract}

This article is freely accessible online.

Correspondence to: Sebastian Szmit, Department of Pulmonary Circulation, Thromboembolic Diseases and Cardiology, Centre of Postgraduate Medical Education, European Health Centre, Borowa 14/18, 05-400 Otwock, Poland. Tel: +48 227103052, Fax: +48 227103169, e-mail: s.szmit@gmail.com

Key Words: Recurrent pericardial effusion, pericardiocentesis, cisplatin, cardio-oncology.
Pericardial diseases constitute an infrequent, although very serious clinical problem among oncological patients. They practically always cause deterioration of the patient's quality of life. The increasing amount of pericardial effusion and the resulting cardiac tamponade is a life-threatening condition which requires urgent diagnostics and treatment.

The European Society of Cardiology (ESC) Position Paper related to cardio-oncology summarizes the knowledge on acute and chronic pericarditis as the complication resulting from anticancer treatment: some cytotoxic drugs and radiotherapy (1). Experts indicate that pericardial complications in oncology are usually associated with mediastinal tumors occupying the pericardium. The document does not discuss the issue of pericardial effusion as a potential manifestation of malignant tumor progression. The present state of knowledge concerning malignant pericarditis is based on several retrospective studies and descriptions of small groups of patients. This article presents our own prospective experience related to the treatment of patients suffering from recurrent pericardial effusion and hemodynamic instability in the course of malignant disease.

The primary purpose of the observation was the assessment of determinants of overall survival of oncological patients subject to emergency pericardiocentesis and intrapericardial chemotherapy with the use of cisplatin due to recurrent symptomatic pericardial effusion and cardiac tamponade.

The detailed aims included the identification of factors which could have direct impact on the overall survival of patients, these included among others: complications of pericardiocentesis and cisplatin administered intrapericardially; signs of inflammatory process: pericardial effusion culture test results, fever, white blood cell count; hemodynamic conditions: baseline level of $\mathrm{N}$-terminal pro-B type natriuretic peptide (NTproBNP), volume and duration of pericardial drainage; interval for recurrence pericardial effusion; possibility of further anticancer treatment. 


\section{Patients and Methods}

The study was performed at the Cardio-Oncological Centre associated with the East European Branch of International Cardioncology Society. This specialized profile of the clinic was decisive in the selection of patients: in the period from March 2012 to October 2017, the hospital admitted patients with diagnosed metastatic cancer diseases whose life was under direct threat due to recurrent pericardial effusion and features of cardiac tamponade. Hemodynamic instability was observed as a result of repetitive occurrence of effusion in the pericardium after a history of pericardiocentesis and the removal of pericardial drainage, or when there was no possibility of removing pericardial drainage because of recurrent hemodynamically significant volume of bloody fluid.

The observation included 17 patients, all subject to emergency pericardiocentesis followed by chemotherapy with the use of cisplatin, which was administered directly to the pericardium. Besides the recurrence of symptomatic pericardial effusion with hemodynamic instability, an additional clinical indication to apply emergency intrapericardial cisplatin therapy was the occurrence of at least one of the following features in every patient: thickening of pericardial plaques suggesting cancerous infiltration, or malignant metastasis into the pericardium; the presence of cancer cells in pericardial fluid sample; newly diagnosed progression of the primary neoplasm confirmed by additional imaging examinations, e.g. computer tomography (CT); lymphangitic carcinomatosis diagnosed based on $\mathrm{CT}$ of the chest.

Pericardiocentesis was performed urgently, under strict echocardiographic (ECG) and fluoroscopic supervision under Hemodynamics Laboratory conditions. Once the pericardium was punctured, a catheter was inserted into the pericardium (pigtail, PeriVac or a central catheter); afterwards the pericardial content was aspirated. Directly after the active drainage was completed, the cytotoxic drug cisplatin was administered pericardially.

Cisplatin was administered through fractionation i.e. a dose of $10 \mathrm{mg}$ diluted in $20 \mathrm{ml}$ of saline was administered daily on subsequent days, directly into the pericardium, while the drainage was being observed. The planned total dosage of cisplatin was $50 \mathrm{mg}$. The therapy was terminated when the fluid had become straw-colored and its drainage had declined to a volume of less than $20 \mathrm{ml} /$ day, then the drain was removed from the pericardium. Extended cisplatin therapy (more than $50 \mathrm{mg}$ ) was used when the fluid was still bloody and its drainage amounted to more than $100 \mathrm{ml} /$ day. All the patients were monitored in the Intensive Care Ward. When pericardial fluid culture test results were positive, additional targeted antibiotic therapy was applied.

Prior to being dismissed from hospital, each patient had been subject to ECG examination in order to assess the amount of fluid in the pericardium. The patients were referred to their original oncological care center, where they were assessed for potential anticancer treatment and the follow-up echocardiographic examinations were performed. Follow-up involved telephone monitoring, during which the patients or their family members were asked to specify: (i) whether there had been recurrent symptoms which should be an indication to perform another pericardiocentesis and pericardial drainage procedure, and if so when that had happened (stating the date), (ii) when the patient had died.

The statistical analyses employed Kaplan-Meier survival estimates and the Cox proportional hazards model. Overall survival was evaluated from the beginning of intrapericardial chemotherapy with cisplatin to date of death.

\section{Results}

The observation encompassed 17 patients (nine women, 53\%), aged 54 years on average (median age 58 years, range from 34 to 68 years). All patients had earlier diagnosis of metastatic cancer; 15 of them had undergone at least one line of chemotherapy and four patients had additionally been subject to radiotherapy. Among oncological diagnoses, the dominant condition was lung cancer (14 patients, 82\%), only three women had other diagnoses: two of them had breast cancer and one colorectal cancer. Adenocarcinoma was the most frequent histopathological diagnosis (11 patients, 64.7\%).

Before pericardiocentesis, all patients had a low performance status: 3 or 4 according to the Eastern Cooperative Oncology Group (ECOG) score.

The mean \pm SD hemoglobin level was $12.9 \pm 2.5 \mathrm{~g} / \mathrm{dl}$, and the median was $12.4 \mathrm{~g} / \mathrm{dl}$ [interquartile range $(\mathrm{IQR})=11.3 \mathrm{~g} / \mathrm{dl}$ and $14.3 \mathrm{~g} / \mathrm{d}$, range=9.1-17.4 g/dl]. Diagnosis of anemia ( $<12 \mathrm{~g} / \mathrm{dl}$ hemoglobin) concerned six patients $(35.3 \%)$. The mean \pm SD white blood cell (WBC) count was $9.2 \pm 4.0 \times 10^{3} / \mu 1$, and the median was $8.1 \times 10^{3} / \mu \mathrm{l} \quad\left(\mathrm{IQR}=6.7-11.8 \times 10^{3} / \mu \mathrm{l}\right.$, range $\left.=2.4-18.3 \times 10^{3} / \mu \mathrm{l}\right)$. This means that seven patients $(41.2 \%)$ had an abnormally high WBC count sufficient to state there was a probability of infection.

Analyzing biomarkers typical of heart failure, it is worth noting that the median level of NTproBNP was $803.5 \mathrm{pg} / \mathrm{ml}$ $(\mathrm{IQR}=190.6-2359 \mathrm{pg} / \mathrm{ml}$, range=78.9-11058 $\mathrm{pg} / \mathrm{ml})$. Moreover, 10 patients $(58.8 \%)$ had NTproBNP level higher than $300 \mathrm{pg} / \mathrm{ml}$, which was suggested as the cut-off value in diagnosis of acute heart failure (2).

In all the patients, the bloody fluid was evacuated. The median volume of the drained fluid for the whole group was $1500 \mathrm{ml}(\mathrm{IQR}=1,200-2,000 \mathrm{ml}$, range=975-6,150 ml). Three patients $(17.6 \%)$ required a cisplatin dosage higher than $50 \mathrm{mg}$. The median drainage time for the whole group was 8 days (IQR $=5-13$ days).

Positive results of pericardial fluid culture tests were noted in three patients and one patient had a positive result for a urine culture test (yeast cultures). Among complications during therapy, the most frequently observed were cardiac arrhythmias in six patients $(35.6 \%)$, and five patients (29.4\%) had fever. The follow-up ECG examinations indicated pericardial constriction in two patients $(11.8 \%)$.

During observation, none of the patients had experienced any symptoms of recurrence of pericardial effusion, which speaks in favor of $100 \%$ efficiency of the applied therapy. Among the observed population, as many as 10 patients (58.8\%) experienced such significant clinical improvement that were sufficiently fit and had adequate cardiopulmonary performance status to be qualify for the subsequent round of anticancer treatment procedures.

In the observed group of 17 patients, 16 died of cancer progression, one remains alive after more than 2 years of 
Table I. Overall survival according to various clinical factors related to pericardiocentesis and pericardial cisplatin therapy.

\begin{tabular}{|c|c|c|c|}
\hline Factor & HR & $95 \% \mathrm{CI}$ & $p$-Value \\
\hline Elevated baseline WBC count $\left(>10^{4} / \mu \mathrm{l}\right)$ & 0.98 & $0.35-2.72$ & 0.97 \\
\hline Baseline anemia defined as hemoglobin $<12 \mathrm{~g} / \mathrm{dl}$ & 0.59 & $0.20-1.73$ & 0.34 \\
\hline NTproBNP> $300 \mathrm{pg} / \mathrm{ml}$ & 0.72 & $0.25-2.1$ & 0.55 \\
\hline Total volume of fluid drained from pericardium $>1,500 \mathrm{ml}$ & 0.84 & $0.29-2.38$ & 0.74 \\
\hline Total pericardial drainage, $>8$ days & 0.55 & $0.19-1.58$ & 0.27 \\
\hline Positive culture test or fever after pericardial cisplatin administration & 1.46 & $0.52-4.03$ & 0.47 \\
\hline Cardiac arrhythmia during intrapericardial cisplatin therapy & 0.66 & $0.23-1.94$ & 0.45 \\
\hline Subsequent systemic anticancer treatment & 0.31 & $0.11-0.9$ & 0.03 \\
\hline
\end{tabular}

WBC: White blood cells; NTproBNP: $N$-terminal pro-B type natriuretic peptide; HR: hazard ratio; CI: confidence interval.

observation. The median survival time for the observed group was 112 days (3.7 months) $[\mathrm{IQR}=45$ days $(1.5$ months)-187 days (more than 6 months)].

The overall survival rate after 1 month was $82.4 \%$ (three patients died within 30 days of observation). The overall survival rate after $2,3,6,9$ and 12 months was $76.5 \%$, $64.7 \%, 29.4 \%, 23.5 \%$, and $11.8 \%$, respectively (two patients lived longer than 1 year).

The overall survival rates were not influenced by hemodynamic parameters such as drainage volume and duration, baseline level of NTproBNP, cardiac arrhythmias related to cisplatin use (Table I). There was no importance of inflammatory markers such as positive culture tests or fever occurring during cisplatin administration, or elevated WBC count $\left(>10^{4} / \mu \mathrm{l}\right)$. A beneficial tendency for longer overall survival was noted when the patient was subject to further anticancer therapy (hazard ratio $=0.31,95 \%$ confidence interval $=0.11-0.9 ; p=0.03$ ).

\section{Discussion}

Malignant disease is one of the most frequent causes of pericardial effusion, which in turn may lead to lifethreatening cardiac tamponade. The presence of pericardial fluid is most often observed in patients with lung cancer and breast cancer, as well as in the course of melanoma, lymphoma and leukemia.

The choice of appropriate treatment depends on the patient's clinical condition. According to the recommendations of the ESC from 2015, related to the diagnosis and treatment of pericardial diseases, when cardiac tamponade is noted, pericardiocentesis is recommended to alleviate the symptoms of the illness and to collect samples for cytological and histopathological tests (recommendation grading: class I, level of evidence B) (3). The aim is the diagnosis of malignant disease as the cause of pericardial effusion. If cancer infiltration of the pericardium is suspected or confirmed, extensive pericardial drainage is recommend in order to prevent recurrent fluid collection, as well as administration of medicines pericardially (recommendation grading: class I, level of evidence B). Pericardial administration of cisplatin should be considered if lung cancer invades the pericardium, and thiotepa in the case of breast cancer metastases (recommendation grading: class IIa, level of evidence B).

Etiology of diagnosis. In patients with such illnesses as pericarditis or pericardial effusion, establishing the etiology is a difficult and controversial issue. Pericardiocentesis is recommended in the case of cardiac tamponade or if bacterial or malignant etiology is suspected (4). Specific clinical characteristics, such as fever, subacute course, inefficiency of anti-inflammatory drugs and the presence of a large amount of fluid or features of cardiac tamponade indicate rather specific causes, mostly related to malignant or infectious etiology (5). In such cases, besides symptomatic and emergency treatment, the management of the primary disease is crucial for further prognosis (6).

One should note that in almost two-thirds of patients with a history of malignant disease, the reason for pericardial fluid collection does not have to be the cancer itself (7). There may be some lesions caused by radiation, after oncological treatment or opportunist infections $(8,9)$. The key test confirming the malignant nature of pericardial disease is the test of fluid from pleural cavities or pericardium, or of the tissue material collected from epicardial biopsy (10).

Spanish authors, based on the observation of 322 patients with moderate or severe pericardial effusion $(40 \%$ patients did not have a defined etiology at the onset of the study), found that cardiac tamponade without the symptoms of any inflammatory condition indicates a high probability of malignant etiology (11). Having observed 150 patients, another study found that cardiac tamponade, during which the bloody fluid is evacuated, indicated either iatrogenic complications (most often resulting from invasive cardiological procedures), or a malignant etiology (12). 
Other data indicate that if a patient with advanced lung cancer experiences pericardial effusion with cardiac tamponade, the most frequent cause is the cancer itself (13).

Polish researchers indicated that malignant diseases are confirmed in most patients requiring pericardiocentesis (14). Unfortunately, cytological testing of the fluid is characterized by low sensitivity, at the level of $46 \%$. Among clinical predictive factors, those typical of malignant diseases seem to include: the presence of cardiac tamponade exponents, enlargement of mediastinal lymph nodes, pericardial thickening observed in imaging examinations, evacuation of bloody fluid from the pericardium and tachycardia higher than $100 \mathrm{bpm}$.

The resulting question that arises is whether one can always administer a cytotoxic drug to the pericardium (15). Our findings confirm that intrapericardial chemotherapy should be preferred, especially in those patients who experience recurrent pericardial effusion accompanied by hemodynamic instability and for whom a malignant etiology is highly likely. According to our evidence, high probability of malignant pericarditis may be assumed not only on the basis of the positive result of cytological examination of the fluid or evident features of pericardium infiltration, but also after taking into consideration that we are faced with the simultaneous progression of cancer or the occurrence of radiological characteristics of lymphangitic carcinomatosis. As far as the aspect of the risk of effusion and cardiac tamponade recurrence is concerned, our treatment proved to be $100 \%$ effective, even in patients with positive inflammatory markers (including positive pericardial fluid culture tests).

Management of the first episode of cardiac tamponade. Pericardiocentesis accompanied by slow discharge of the fluid (up to $30 \mathrm{ml} / 24 \mathrm{~h}$ ) should be favorable for the adhesion of pericardial plaque, and thus should prevent further accumulation of the fluid. Specialist literature does not provide sufficient data, which would confirm the efficacy of such a procedure (16).

In order to prevent the accumulation of the fluid, one can administer sclerotherapeutic or cytotoxic drugs intrapericardially (17). The drugs administered intrapericardially should be selected depending on the type of cancer (18). Thiotepa has been proven to be effective in the case of intrapericardial metastases of breast cancer $(19,20)$.

In a randomized study of 79 patients with various cancer types who had experienced pericardial effusion, an extended drainage procedure was compared to bleomycin therapy (15 $\mathrm{mg}$ on the first day, then $10 \mathrm{mg}$ every 48 hours) (20). Bleomycin acts as a sclerotherapeutic and anticancer agent. The results, however, were not satisfactory because the lack of effusion recurrence after 2 months was noted in $29 \%$ and $46 \%$ patients, respectively; the difference was statistically insignificant. The median overall survival rates were 79 days and 119 days, respectively.

Cisplatin seems to be the medicine of choice in the case of pericardium being invaded by lung cancer. Maisch et al. also indicated the efficiency of cisplatin administered intrapericardially: more than $85 \%$ patients were free from fluid recurrence for at least 3 months. The authors draw attention to the fact that over 20 years among 357 patients subject to pericardiocentesis, 68 suffered from pericardial effusion related to oncological problems: 42 had malignant pericarditis, 15 experienced effusion following radiotherapy, and five were from other causes, e.g. resulting from the course of potential viral infection and in the course of immunological reaction. This is why the authors pointed to the urgent need for defining the etiology of effusion (22). In a register of 42 patients with malignant pericardial effusion, cisplatin administered intrapericardially (30 mg) was less effective in patients with breast cancer in comparison with those with lung cancer. In the 6 months of observation, recurrence of effusion was found in $37.5 \%$ of those with breast cancer and in only $4.5 \%$ of those with lung cancer, a difference that was statistically significant (23).

Tomkowski and Filipecki indicated the efficiency of intrapericardial cisplatin administration in 15 out of 16 patients $(93.75 \%)$ among lung cancer patients with malignant pericardial effusion whose survival time was at least 30 days. The total survival of patients ranged from 2 to 24.1 months (the median being 3.7 months, and the average being 6.59 months). Paroxysmal atrial fibrillation was diagnosed in $18.8 \%$ patients, and one patient suffered from nausea (24).

Bischiniotis et al. published a study involving 25 patients with adenocarcinoma of the lung. The authors assumed that this histological type was usually accompanied by the risk of pericardial effusion. All the patients subject to analysis underwent pericardiocentesis due to cardiac tamponade. Cisplatin was administered according to the following regime: $10 \mathrm{mg}$ in $20 \mathrm{ml}$ for 3 days. In $80 \%$ of the cases, the fluid was bloody, and its volume ranged from 350 to $1,700 \mathrm{ml}$ (median=750 ml). Survival time ranged from 3 to 92 weeks (4.5 months on average). Respiratory failure was the cause of death. The authors assessed therapy safety, and out of 25 patients observed paroxysmal atrial fibrillation in three $(12 \%)$, non-sustained ventricular tachycardia in two $(8 \%)$, and thickening of the pericardium without constriction in four $(16 \%)(25)$.

One multicenter study analyzed a group of 119 patients and compared the efficacy of four therapeutic strategies: (i) extended drainage procedure, (ii) systemic chemotherapy, (iii) intrapericardial chemotherapy, (iv) combination of intrapericardial and systemic chemotherapy. A complete response was found in 37 out of 53 patients $(70 \%)$ in the combination chemotherapy, 12 out of 22 patients $(54.5 \%)$ in intrapericardial chemotherapy, in five out of 27 patients 
$(18.5 \%)$ in systemic chemotherapy, and in four out of 17 patients $(23.5 \%)$ after drainage procedure - the differences were statistically significant $(p<0.001)$. Finally, the best survival time was observed among patients subject to combination chemotherapy - as many as 12 out of 53 patients $(23 \%)$ lived longer than 12 months. Cisplatin administered intrapericardially was effective in $98 \%$ of the cases (other medications only in 80\%). The authors concluded that intrapericardial chemotherapy provided good control of pericarditis in $92 \%$ of cases, and a complete resolution in $65 \%$ (26). Our current study confirms that intrapericardial chemotherapy may be similarly effective for recurrent pericardial effusion in patients with hemodynamic instability and not only in patients with lung cancer or adenocarcinoma.

Treatment of recurrent pericardial effusion. Malignant pericardial effusion may present various volumes, including the condition of a threat of tamponade which is often recurrent (27). The ESC recommends that in order to prevent recurrent accumulation of malignant effusion in the pericardium, one may consider percutaneus balloon pericardiotomy (recommendation grading: class IIb, level of evidence B). Percutaneus balloon pericardiotomy enables the execution of a channel between the pericardium cavity and pleural cavity, which facilitates pericardial effusion drainage. Such procedure seems to be effective in patients with vast amounts of pericardial fluids and recurrent symptoms of cardiac tamponade.

Based on the revision of 31 studies related to prognoses, it has been estimated that the recurrence of malignant pericardial effusion is evident in $38.3 \%$ patients after pericardiocentesis alone, in $12.1 \%$ patients after pericardiocentesis with extended drainage procedure, in $10.8 \%$ patients after intrapericardial administration of sclerotising medications and in $10.3 \%$ patients after percutaneous pericardiotomy. The efficiency of percutaneus balloon pericardiotomy seems to be highest; however, the difference was not found to be statistically significant. The incidence of recurrent pericardial effusion after percutaneus balloon pericardiotomy in comparison with classical pericardiocentesis was $7.4 \%$ in comparison to $14.3 \%$ $(p=0.48)$, the percentage of the occurrence of complications being comparable ( 7\%) (28).

At the Mayo Clinic Rochester, an analysis of treatment efficiency of the first and the subsequent episodes of malignant pericardial effusion by means of drainage was performed (29). The data for the period of ca. 20 years were retrospectively collected. Among the 1002 pericardiocentesis procedures performed, 275 referred to oncological patients. The recurrence of pericardial effusion or an extended drainage requiring a repeated therapy was noted for 59 patients: (i) in 43 out of 118 patients (36\%) who underwent pericardiocentesis only, (ii) in 16 out of 139 patients $(11.5 \%)$ after pericardiocentesis with extended drainage, (iii) in none of 18 patients after surgical procedure. Recurrent pericardial effusion was significantly related to the following independent risk factors: large fluid amount and urgent treatment of the first episode as well as no application of extended drainage. Recurrence following the second intervention was observed in 12 patients: 11 required extended drainage, one had to be subject to a surgical procedure. The median overall survival was 135 days and, crucially, $26 \%$ of the patients survived for at least 12 months. Independent factors with a negative impact on survival rates were: male gender, the presence of malignant cells in the fluid, diagnosis of lung cancer, the characteristics of cardiac tamponade, and unstable hemodynamics.

Only one publication provides assessment of cisplatin effectiveness in the treatment of recurrent effusion in the pericardium (30). The presented analysis encompassed 46 patients ( $89 \%$ with thoracic malignancies), 35 of whom underwent pericardiocentesis, and 11 video-assisted thoracoscopic surgery procedure. Recurrent pericardial effusion was defined as the presence of more than $50 \mathrm{ml}$ of fluid per day during pericardial drainage performed for the subsequent 5 to 7 days. In the observed group, $82.6 \%$ patients lived at least 30 days. Cisplatin was effective in 43 out of $46(93.5 \%)$ observed patients and in 35 out of 38 $(92 \%)$ patients who survived at least 30 days. The median survival time for this subgroup of 38 patients was 102.5 days. It is worth remembering that this analysis excluded patients who died within 30 days. The study also analyzed therapy safety: atrial fibrillation occurred in seven out of 46 patients $(15.2 \%)$, and sclerotization of the pericardium, although without constrictions, was revealed in five out of 46 patients $(10.9 \%)$.

Cardiovascular and coagulation conditions may affect overall survival in patients with cancer (31-33). Therefore, in our patients, the worst prognosis should be expected with more severe symptoms of hemodynamic instability and aggravated inflammatory process. Numico et al., looking at their own observations, suggested that patients with greater volume of effusion may have a better response after intrapericardial therapy (34). On the other hand, evidence of tamponade on ECG, larger effusion volume, and positive cytological findings may predict a higher risk of paradoxical hemodynamic instability after pericardial window in patients with cancer (35). Our study revealed that the degree of hemodynamic instability was insignificant for the final efficacy of rescue intrapericardial cisplatin chemotherapy.

A subsequent episode of pericardial effusion should have a higher mortality risk. One should bear in mind that in the first episode of pericardial effusion, the ESC recommends that in the case of confirmed diagnosis of a malignant disease, a patient should qualify for systematic anticancer treatment (recommendation grading: class I, level of 
evidence B). It is crucial that a worse prognosis is likely to occur in those patients who do not receive later systemic anticancer therapy. There are no precise data available from literature stating how many oncological patients after diagnosis of cardiac tamponade are subsequently treated systemically for malignant disease.

Our observation of this group of 17 patients confirms, first of all that intrapericardial cisplatin therapy should be taken into consideration as early as possible during the first episode of cardiac tamponade, but it may also be highly effective in recurrent pericardial effusion with hemodynamic complications. The overall survival rates in our population were higher than (or at least similar to) those in the studies where cisplatin was administered directly after the first cardiac tamponade episode. What is crucial is that we did not exclude from our analyses the patients who died within 30 days. Secondly, the use of daily fractionated doses of cisplatin seems to be a very effective and safe procedure. Thirdly, pericardiectomy, pericardial window, or percutaneus balloon pericardiotomy may be equally effective in patients with recurrent pericardial effusion, but it is surely more devastating than emergency pericardiocentesis and intrapericardial cisplatin therapy that we suggest (36). It is unlikely that after such a surgical procedure an oncological patient would be capable of undertaking further systemic anticancer treatment, whereas the majority of our patients were in such good physical condition that in their further treatment they were able to receive chemotherapy and had a chance of significantly longer survival.

\section{Conflicts of Interest}

The Authors declare that they have no conflict of interest in regard to this study.

\section{Informed Consent}

Informed consent was obtained from all individual participants included in the study.

\section{References}

1 Zamorano JL, Lancellotti P, Munoz D, Aboyans V, Asteggiano R, Galderisi M, Habib G, Lenihan DJ, Lip GYH, Lyon AR, Fernandez TL, Mohty D, Piepoli MF, Tamargo J, Torbicki A, Suter TM, Achenbach S, Agewall S, Badimon L, BaronEsquivias G, Baumgartner H, Bax JJ, Bueno H, Carerj S, Dean V, Erol C, Fitzsimons D, Gaemperli O, Kirchhof P, Kolh P, Nihoyannopoulos P, Ponikowski P, Roffi M, Carneiro AV, Windecker S, Minotti G, Cardinale D, de Azambuja E, Dent S, Ewer MS, Farmakis D, Fietkau R, McGale P and Ringwald J: 2016 ESC Position Paper on Cancer Treatments and Cardiovascular Toxicity developed under the auspices of the ESC committee for practice guidelines, the Task Force for Cancer Treatments and Cardiovascular Toxicity of the European Society of Cardiology (ESC). Eur J Heart Fail 19(1): 9-42, 2017.
2 Ponikowski P, Voors AA, Anker SD, Bueno H, Cleland JGF, Coats AJS, Falk V, Gonzalez-Juanatey JR, Harjola VP, Jankowska EA, Jessup M, Linde C, Nihoyannopoulos P, Parissis JT, Pieske B, Riley JP, Rosano GMC, Ruilope LM, Ruschitzka F, Rutten FH, van der Meer P, Filippatos G, McMurray JJV, Aboyans V, Achenbach S, Agewall S, Al-Attar N, Atherton JJ, Bauersachs J, Camm AJ, Carerj S, Ceconi C, Coca A, Elliott P, Erol C, Ezekowitz J, Fernandez-Golfin C, Fitzsimons D and Guazzi M: 2016 ESC Guidelines for the Diagnosis and Treatment of Acute and Chronic Heart Failure: The Task Force for the Diagnosis and Treatment of Acute and Chronic Heart Failure of the European Society of Cardiology (ESC). Developed with the special contribution of the Heart Failure Association (HFA) of the ESC. Eur J Heart Fail 18(8): 891-975, 2016.

3 Adler Y, Charron P, Imazio M, Badano L, Baron-Esquivias G, Bogaert J, Brucato A, Gueret P, Klingel K, Lionis C, Maisch B, Mayosi B, Pavie A, Ristic AD, Tenas MS, Seferovic P, Swedberg K and Tomkowski W: 2015 ESC Guidelines for the Diagnosis and Management of Pericardial Diseases, the Task Force for the Diagnosis and Management of Pericardial Diseases of the European Society of cardiology (ESC) endorsed by: The european association for cardio-thoracic Surgery (EACTS). Eur Heart J 36(42): 2921-2964, 2015.

4 Imazio $\mathrm{M}$ and Adler Y: Management of pericardial effusion. Eur Heart J 34(16): 1186-1197, 2013.

5 Imazio M, Cecchi E, Demichelis B, Ierna S, Demarie D, Ghisio A, Pomari F, Coda L, Belli R and Trinchero R: Indicators of poor prognosis of acute pericarditis. Circulation 115(21): 27392744, 2007.

6 Imazio M, Mayosi BM, Brucato A, Markel G, Trinchero R, Spodick DH and Adler Y: Triage and management of pericardial effusion. J Cardiovasc Med 11(12): 928-935, 2010.

7 Porte HL, Janecki-Delebecq TJ, Finzi L, Metois DG, Millaire A and Wurtz AJ: Pericardoscopy for primary management of pericardial effusion in cancer patients. Eur J Cardiothorac Surg 16(3): 287-291, 1999.

8 Vaitkus PT, Herrmann HC and Lewinter MM: Treatment of malignant pericardial-effusion. JAMA 272(1): 59-64, 1994.

9 Tamari K, Isohashi F, Akino Y, Suzuki O, Seo Y, Yoshioka Y, Hayashi Y, Nishida T, Takehara T, Mori M, Doki Y and Ogawa $\mathrm{K}$ : Risk factors for pericardial effusion in patients with stage $\mathrm{i}$ esophageal cancer treated with chemoradiotherapy. Anticancer Res 34(12): 7389-7393, 2014.

10 Meyers DG and Bouska DJ: Diagnostic usefulness of pericardial fluid cytology. Chest 95(5): 1142-1143, 1989.

11 Sagrista-Sauleda J, Merce J, Permanyer-Miralda G and SolerSoler J: Clinical clues to the causes of large pericardial effusions. Am J Med 109(2): 95-101, 2000.

12 Atar S, Chiu J, Forrester JS and Siegel RJ: Bloody pericardial effusion in patients with cardiac tamponade - is the cause cancerous, tuberculous, or latrogenic in the 1990s? Chest 116(6): 1564-1569, 1999.

13 Wang PC, Yang KY, Chao JY, Liu JM, Perng RP and Yen SH: Prognostic role of pericardial fluid cytology in cardiac tamponade associated with non-small cell lung cancer. Chest 118(3): 744-749, 2000.

14 Pawlak-Cieslik A, Szturmowicz M, Fijalkowska A, Gatarek J, Gralec R, Blasinska-Przerwa K, Szczepulska-Wojcik E, Skoczylas A, Bilska A and Tomkowski W: Diagnosis of 
malignant pericarditis: A single-centre experience. Kardiol Pol 70(11): 1147-1153, 2012.

15 Lestuzzi C, Lafaras C, Bearz A, Gralec R, Viel E, Buonadonna $\mathrm{A}$ and Bischiniotis $\mathrm{T}$ : Malignant pericardial effusion: Sclerotherapy or local chemotherapy? Br J Cancer 101(4): 734735, 2009.

16 Ristic AD, Imazio M, Adler Y, Anastasakis A, Badano LP, Brucato A, Caforio ALP, Dubourg O, Elliott P, Gimeno J, Helio T, Klingel K, Linhart A, Maisch B, Mayosi B, Mogensen J, Pinto Y, Seggewiss H, Seferovic PM, Tavazzi L, Tomkowski W and Charron P: Triage strategy for urgent management of cardiac tamponade: A Position Statement of the European Society of Cardiology Working Group on Myocardial and Pericardial Diseases. Eur Heart J 35(34): 2279-2284, 2014.

17 Girardi LN, Ginsberg RJ and Burt ME: Pericardiocentesis and intrapericardial sclerosis: Effective therapy for malignant pericardial effusions. Ann Thorac Surg 64(5): 1422-1427, 1997.

18 Martinoni A, Cipolla CM, Civelli M, Cardinale D, Lamantia G, Colleoni M, DeBraud F, Susini G, Martinelli G, Goldhirsh A and Fiorentini C: Intrapericardial treatment of neoplastic pericardial effusions. Herz 25(8): 787-793, 2000.

19 Bishiniotis TS, Antoniadou S, Katseas G, Mouratidou D, Litos $\mathrm{AG}$ and Balamoutsos $\mathrm{N}$ : Malignant cardiac tamponade in women with breast cancer treated by pericardiocentesis and intrapericardial administration of triethylenethiophosphoramide (thiotepa). Am J Cardiol 86(3): 362-+, 2000.

20 Colleoni M, Martinelli G, Beretta F, Marone C, Gallino A, Fontana M, Graffeo R, Zampino G, De Pas T, Cipolla G, Martinoni $\mathrm{C}$ and Goldhirsch A: Intracavitary chemotherapy with thiotepa in malignant pericardial effusions: An active and welltolerated regimen. J Clin Oncol 16(7): 2371-2376, 1998.

21 Kunitoh H, Tamura T, Shibata T, Imai M, Nishiwaki Y, Nishio M, Yokoyama A, Watanabe K, Noda K, Saijo N and Grp JLCS: A randomised trial of intrapericardial bleomycin for malignant pericardial effusion with lung cancer (JCOG9811). Br J Cancer 100(3): 464-469, 2009.

22 Maisch B, Ristic A and Pankuweit S: Evaluation and management of pericardial effusion in patients with neoplastic disease. Prog Cardiovasc Dis 53(2): 157-163, 2010.

23 Maisch B, Ristic AD, Pankuweit S, Neubauer A and Moll R: Neoplastic pericardial effusion - efficacy and safety of intrapericardial treatment with cisplatin. Eur Heart J 23(20): 1625-1631, 2002.

24 Tomkowski WZ and Filipecki S: Intrapericardial cisplatin for the management of patients with large malignant pericardial effusion in the course of the lung cancer. Lung Cancer 16(2-3): 215-222, 1997.

25 Bischiniotis TS, Lafaras CT, Platogiannis DN, Moldovan L, Barbetakis NG, Katseas GP: Intrapericardial cisplatin administration after pericardiocentesis in patients with lung adenocarcinoma and malignant cardiac tamponade. Hellenic $\mathbf{J}$ Cardiol 46(5): 324-9, 2005.

26 Lestuzzi C, Bearz A, Lafaras C, Gralec R, Cervesato E, Tomkowski W, DeBiasio M, Viel E, Bishiniotis T, Platogiannis DN, Buonadonna A, Tartuferi L, Piazza R, Tumolo S, Berretta $\mathrm{M}$, Santini $\mathrm{F}$ and Imazio $\mathrm{M}$ : Neoplastic pericardial disease in lung cancer: Impact on outcomes of different treatment strategies. A multicenter study. Lung Cancer 72(3): 340-347, 2011.
27 Imazi M, Demichelis B, Parrini I, Favro E, Beqaraj F, Cecchi E, Pomari F, Demarie D, Ghisio A, Belli R, Bobbio M and Trinchero R: Relation of acute pericardial disease to malignancy. Am J Cardiol 95(11): 1393-1394, 2005.

28 Swanson N, Mirza I, Wijesinghe N and Devlin G: Primary percutaneous balloon pericardiotomy for malignant pericardial effusion. Catheter Cardiovasc Interv 71(4): 504-507, 2008.

29 Tsang TSM, Seward JB, Barnes ME, Bailey KR, Sinak LJ, Urban LH and Hayes SN: Outcomes of primary and secondary treatment of pericardial effusion in patients with malignancy. Mayo Clin Proc 75(3): 248-253, 2000.

30 Tomkowski WZ, Wisniewska J, Szturmowicz M, Kuca P, Burakowski J, Kober $\mathrm{J}$ and Fijalkowska A: Evaluation of intrapericardial cisplatin administration in cases with recurrent malignant pericardial effusion and cardiac tamponade. Support Care Cancer 12(1): 53-57, 2004.

31 Jurczak W, Szmit S, Sobocinski M, Machaczka M, DrozdSokolowska J, Joks M, Dzietczenia J, Wrobel T, Kumiega B, Zaucha JM, Knopinska-Posluszny W, Spychalowicz W, Prochwicz A, Drohomirecka A and Skotnicki AB: Premature cardiovascular mortality in lymphoma patients treated with (R)CHOP regimen - a national multicenter study. Int J Cardiol 168(6): 5212-5217, 2013

32 Szmit S, Langiewicz P, Zolnierek J, Nurzynski P, Zaborowska M, Filipiak KJ, Opolski G and Szczylik C: Hypertension as a predictive factor for survival outcomes in patients with metastatic renal cell carcinoma treated with sunitinib after progression on cytokines. Kidney Blood Press Res 35(1): 18-25, 2012.

33 Zaborowska-Szmit M, Kowalski DM, Piorek A, Krzakowski M and Szmit S: A decrease in D-dimer concentration and an occurrence of skin rash as iatrogenic events and complementary predictors of survival in lung cancer patients treated with EGFR tyrosine kinase inhibitors. Pharmacol Rep 68(6): 1140-1148, 2016.

34 Numico G, Cristofano A, Occelli M, Sicuro M, Mozzicafreddo A, Fea E, Colantonio I, Merlano M, Piovano P and Silvestris N: Prolonged drainage and intrapericardial bleomycin administration for cardiac tamponade secondary to cancerrelated pericardial effusion. Medicine 95(15), 2016.

35 Wagner PL, McAleer E, Stillwell E, Bott M, Rusch VW, Schaffer W and Huang J: Pericardial effusions in the cancer population: Prognostic factors after pericardial window and the impact of paradoxical hemodynamic instability. $\mathrm{J}$ Thorac Cardiovasc Surg 141(1): 34-38, 2011.

36 Imazio M, Spodick DH, Brucato A, Trinchero R and Adler Y: Controversial issues in the management of pericardial diseases. Circulation 121(7): 916-U128, 2010.
Received November 24, 2017

Revised December 17, 2017

Accepted December 21, 2018 